\title{
A potential role for a genetic variation of AKAP5 in human aggression and anger control
}

\section{Sylvia Richter ${ }^{1,2,3}$, Xenia Gorny ${ }^{2}$, Josep Marco-Pallares ${ }^{4}$, Ulrike M. Krämer $^{5}$, Judith Machts ${ }^{6}$, Adriana Barman ${ }^{2}$, Hans-Gert Bernstein ${ }^{7}$, Rebecca Schüle ${ }^{8}$, Ludger Schöls ${ }^{8,9}$, Antoni Rodriguez-Fornells $^{4,10}$, Carsten Reissner ${ }^{11}$, Torsten Wüstenberg ${ }^{12}$, Hans-Jochen Heinze ${ }^{2,3,6}$, Eckart D. Gundelfinger ${ }^{2}$, Emrah Düzel ${ }^{3,6,13,14}$, Thomas F. Münte ${ }^{5}$, Constanze I. Seidenbecher ${ }^{2}$ and Björn H. Schott ${ }^{2,6,12 *}$}

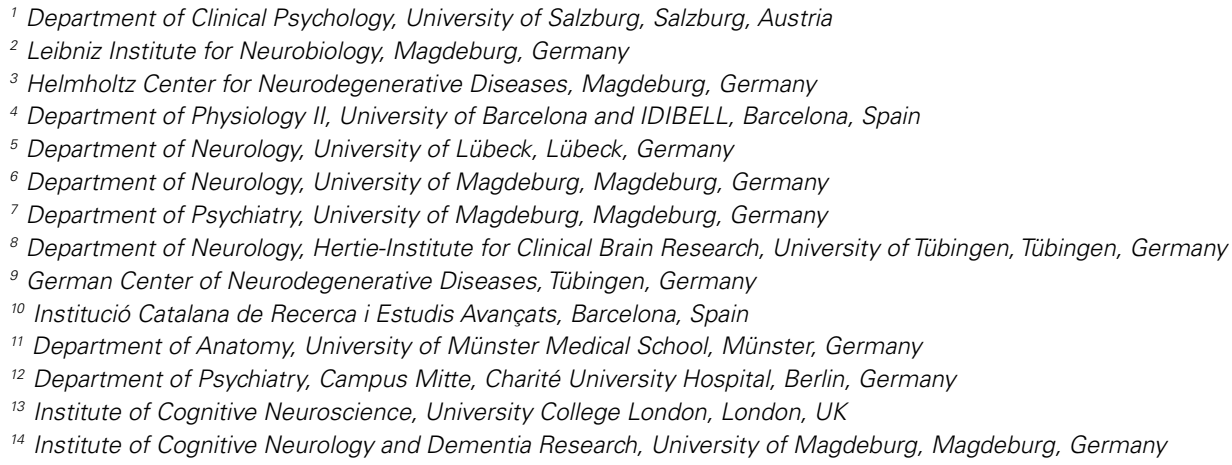

Edited by:

Chad Edward Forbes, University of Delaware, USA

Reviewed by:

Lutz Jäncke, University of Zurich, Switzerland

Ruthger Righart, Institute for Stroke and Dementia Research, Germany

\section{*Correspondence.}

Björn H. Schott, Leibniz Institute for Neurobiology, Brenneckestr. 6, 39118 Magdeburg, Germany.

e-mail: bschott@neuro2.med.

uni-magdeburg.de
The A-kinase-anchoring protein 5 (AKAP5), a post-synaptic multi-adaptor molecule that binds G-protein-coupled receptors and intracellular signaling molecules has been implicated in emotional processing in rodents, but its role in human emotion and behavior is up to now still not quite clear. Here, we report an association of individual differences in aggressive behavior and anger expression with a functional genetic polymorphism (Pro100Leu) in the human AKAP5 gene. Among a cohort of 527 young, healthy individuals, carriers of the less common Leu allele (15.6\% allele frequency) scored significantly lower in the physical aggression domain of the Buss and Perry Aggression Questionnaire and higher in the anger control dimension of the state-trait anger expression inventory. In a functional magnetic resonance imaging experiment we could further demonstrate that AKAP5 Pro100Leu modulates the interaction of negative emotional processing and executive functions. In order to investigate implicit processes of anger control, we used the well-known flanker task to evoke processes of action monitoring and error processing and added taskirrelevant neutral or angry faces in the background of the flanker stimuli. In line with our predictions, Leu carriers showed increased activation of the anterior cingulate cortex (ACC) during emotional interference, which in turn predicted shorter reaction times and might be related to stronger control of emotional interference. Conversely, Pro homozygotes exhibited increased orbitofrontal cortex (OFC) activation during emotional interference, with no behavioral advantage. Immunohistochemistry revealed AKAP5 expression in post mortem human ACC and OFC. Our results suggest that AKAP5 Pro100Leu contributes to individual differences in human aggression and anger control. Further research is warranted to explore the detailed role of AKAP5 and its gene product in human emotion processing.

Keywords: AKAP5, genetic, aggression, anger, fMRI

\section{INTRODUCTION}

Human aggression shows considerable interindividual variability. Significant contributions to aggression originate in the emotion of anger, which itself shows high variability within the population (Berkowitz and Harmon-Jones, 2004). Several studies suggest that aggressive behavior is related to interactions of environmental factors like aversive childhood experience or substancerelated disorders with genetic variations in monoaminergic neuromodulatory systems, specifically dopaminergic, noradrenergic, and serotonergic neurotransmission, might influence aggressive behavior (Caspi et al., 2002; Panksepp, 2006; Kang et al., 2008; Kulikova et al., 2008; Heinz et al., 2011). Functional neuroimaging studies have demonstrated that genetic variants linked to aggression and anger are associated with altered neuronal activation patterns during emotional processing (Meyer-Lindenberg et al., 2006; Buckholtz and Meyer-Lindenberg, 2008). Up to now, genetic studies on anger and aggression have focused on variants directly related to these transmitter systems, like receptors or metabolizing 
enzymes (Buckholtz and Meyer-Lindenberg, 2008; Kang et al., 2008; Kulikova et al., 2008; Hess et al., 2009). G-protein-coupled receptor (GPCR) activation by monoamines, however, triggers complex intracellular signaling cascades that exert relatively longlasting influences on neuronal processing. Thus the question arises whether genetic heterogeneity in these signaling cascades might also influence interindividual variability in human anger and aggression. Initial evidence from depressed patients suggests that the transcription factor CREB, which is activated by GPCR signaling, shows genetic variations that affect human anger expression (Perlis et al., 2007), but the influence of genetic variations in intracellular signaling molecules on anger and aggression in the healthy population remains thus far unclear.

The A-kinase-anchoring proteins (AKAPs) are a family of proteins that bind the cAMP-dependent protein kinase A (PKA), a major effector enzyme of numerous GPCRs. AKAPs are involved in the subcellular targeting of receptor-activated protein kinases and phosphatases and are likely to play a crucial role in the coordination of GPCR-mediated signaling (Carnegie et al., 2009). AKAP5 (AKAP79/150) is a multi-adaptor molecule that binds GPCRs, particularly beta-adrenergic receptors (Fraser et al., 2000; Gardner et al., 2007), protein kinases A and C, protein phosphatase 2B, and MAGuK-type post-synaptic adaptor molecules (Dell'Acqua et al., 2006; Figure 1A).

In striatal and prefrontal cortex neurons, where precise communication between glutamatergic and dopaminergic systems is crucial to neural processes underlying cognitive control, emotion, and reward, the AKAP5 gene product was shown to be critically involved in this crosstalk between D1 receptor-dependent neuromodulation and AMPA receptor-mediated synaptic plasticity (Swayze et al., 2004). Animal research has provided converging evidence for a role of AKAP5 in the neural processing of (negative) emotions. In rodents, inhibition of the corresponding protein AKAP150 has been associated with impaired consolidation of fear learning (Moita et al., 2002), specifically with reduced consolidation and enhanced extinction of fear memories (Nijholt et al., 2008). Disruption of AKAP5 in mice has been associated with physiological and behavioral abnormalities, including deficits in long-term depression (LTD) and memory retention (Tunquist et al., 2008).

So far, the impact of genetic variations in AKAP5 on human behavior is yet unclear. In the human AKAP5 gene (Chr 14q21-24), a functional genetic polymorphism leads to a substitution of proline to leucine at position 100 of the protein (AKAP5 Pro100Leu; dbSNP rs2230491). Protein structure prediction suggests that this amino acid substitution might influence protein folding and curvature (Figure 1B) Based on cell-biological findings and animal experiments about AKAP function as a key synchronizer of neuronal events (Tunquist et al., 2008) and its association with GPCR signaling, we therefore hypothesized that the polymorphism might influence human aggression as well as aggression-related emotions and their control. Previous expression studies of the human AKAP5 gene product (AKAP79) showed a high abundance in several CNS regions relevant for emotional and motivational processes, including the amygdala, the hippocampus and the striatum (Sik et al., 2000; Ulfig et al., 2001, 2003), also pointing to a role of AKAP5 in human emotional processing.

We investigated the effects of AKAP5 Pro100Leu on human aggressive behavior and anger expression in young, healthy participants by using well-established questionnaires of anger and aggression (Buss and Perry, 1992; State Trait Anger Expression Inventory; Spielberger, 1991). Based on the questionnaire data,
A

B

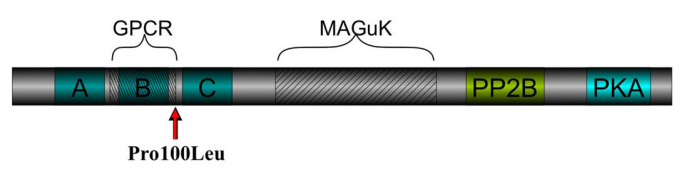

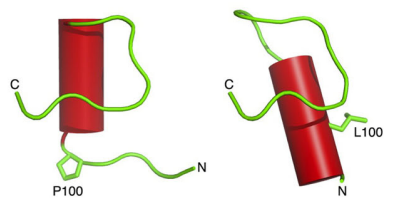

D

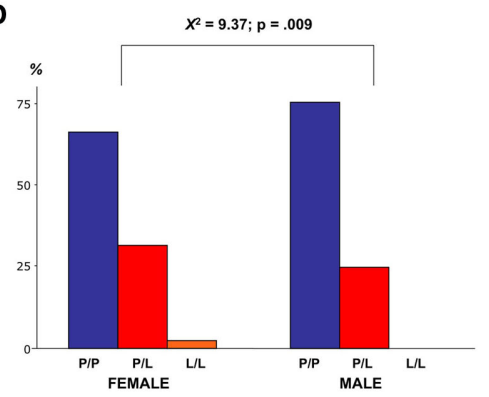

FIGURE 1 | (A) Structure of the human AKAP5 protein with the Pro100Leu polymorphism. A, B, C, basic membrane association domains; $\mathrm{PP} 2 \mathrm{~B}$, protein phosphatase $2 \mathrm{~B}$ binding site; PKA, protein kinase $\mathrm{A}$ binding site; GPCR, G-protein-coupled receptor binding region; MAGuK, binding region for membrane-associated guanylate kinase proteins. (B) Protein structure prediction using PEP-FOLD software suggests that AKAP5 Pro100Leu substitution leads to extension of an $\alpha$-helical region (depicted as red cylinder), with potential consequences on the folding of domains located downstream of the mutation. (C) Genotyping of the AKAP5 Pro100Leu polymorphism. Alul digestion of the PCR products results in two fragments $(174+98 \mathrm{bp})$ for the Leu allele or a single fragment (272 bp) for the Pro allele. PP, Pro/Pro; PL, Pro/Leu; LL, Leu/Leu; M, DNA size marker. (D) Gender bias in the distribution of the AKAP5 Pro100Leu polymorphism. Bar plots depict percentages of Pro/Pro (P/P), Pro/Leu $(P / L)$, and Leu/Leu (L/L) carriers, separated by gender. The Leu allele was significantly more prevalent among women. 
which revealed significantly lower physical aggression and significantly higher anger-control behavior in carriers of the more rare Leu allele, we developed a functional magnetic resonance imaging (fMRI) study to investigate the neurobiological processes that may underlie these behavioral effects. Because Leu carriers exhibited stronger anger control, we aimed to integrate anger-related stimuli in a demanding cognitive task.

Several studies of emotional conflict regulation haved use an "emotional Stroop" task (see Etkin et al., 2006; Haas et al., 2006; Egner et al., 2008) and discussed the important role of rostral and caudal anterior cingulate cortex (ACC) in the processing of incompatible emotional dimensions. The cited emotional Stroop tasks have used compatible and incompatible combinations of emotional words and emotional faces in order to create congruent and incongruent trials and to provoke "real" emotional conflicts in incongruent conditions. In another version of the emotional Stroop task, subjects are asked to identify the ink color of neutral vs. emotionally salient words. The slower reaction times (RTs) for emotionally salient words have been suggested to reflect emotional interference (Etkin et al., 2006). Concerning the specific emotion of anger, Van Honk et al. (2001) demonstrated that individuals scoring high on trait-anger exhibited an attentional bias toward angry faces. In addition to Stroop tasks, variants of the Flanker task (Eriksen and Eriksen, 1974) have also been used to investigate the interaction of emotional and cognitive processes. Neuroimaging studies of classical Flanker tasks typically discuss the role of the dorsal anterior cingulate cortex (dACC) in conflict processing during incongruent trials (Kerns et al., 2004; Mansouri et al., 2009). In emotional variants of this paradigm, when neutral vs. emotional faces were used as target stimuli (Fenske and Eastwood, 2003; Horstmann et al., 2006), prolonged RTs have been observed for the processing of negative emotional stimuli. It should be noted, though, that in all these tasks, processing of the emotional stimuli was directly relevant for task performance.

An alternative approach was used in an ERP-Study by Wiswede et al. (2009), where pictures from the International Affective Picture System (IAPS) served as task-irrelevant distracters during a flanker paradigm. A stronger increase of the error-related negativity (ERN) was observed for errors in trials with negative emotional distraction (Wiswede et al., 2009). It should be noted though, that no neuroimaging study so far has used a comparable paradigm (i.e., emotional task-irrelevant distracters in the background of flanker stimuli) with face stimuli.

Given our primary aim, to assess neural mechanisms underlying the genetically mediated individual differences in anger control, we aimed to design a paradigm in which emotional stimuli were anger-related and interfered with task performance. To this end, we designed a modified version of the task described by Wiswede et al. (2009). Instead of IAPS pictures, facial emotional distracters (neutral vs. angry) were displayed in the background of the flanker stimuli in order to assess, how carriers of the two alleles might differ in their neural processing of interference of attention and anger processing rather than processing of negative emotions in general. We hypothesized that Leu carriers, who exhibit stronger anger control, would show a higher ability to successfully focus on the cognitive flanker task while fading out the emotional distracters. At a neural level, such a behavioral advantage might be associated with increased ACC activation. Pro homozygotes, in contrast, might react more intensely to the emotional distracters, which might be reflected by weaker task performance and neuronal activation in emotion-relevant prefrontal areas like the orbitofrontal cortex (OFC).

Since the AKAP5 expression pattern in human cortical areas implicated in the cognitive control of emotions, has not been published so far, we also performed post mortem immunohistochemistry in the frontolimbic cortical regions of interest (ROIs) of our fMRI study.

\section{MATERIALS AND METHODS PARTICIPANTS}

Five hundred fifty-seven young (age range 18-36 years, mean $22.9 \pm 3.1$ ), healthy volunteers participated in the behavioral part of our study. All participants had at least obtained university entrance diploma or were enrolled at the Otto von Guericke University Magdeburg or at the Magdeburg-Stendal University of Applied Sciences. The university entrance diploma was selected as one of our criteria of inclusion in order to reach a comparable educational background within our sample. Furthermore, the participants were recruited from all departments of both universities like medicine, natural sciences, educational sciences, social and health sciences, or economic sciences which ensured a certain degree of variance in the sample. Moreover, students of psychology were only allowed to take part in the study within their first academic year, to make sure, that they were as naïve as the other participants to our questionnaires and tests. In addition to the analyses of our data, a post hoc analysis of AKAP5 Pro100Leu effects on Buss-Perry aggression questionnaire (BPAQ) scores was performed in a cohort of 604 participants from the University of Barcelona community (457 female, mean age $21.6 \pm 3.1$ years; see Table 3). This cohort had been described in detail previously (Krämer et al., 2007).

Seventy young, healthy German volunteers (35 Pro homozygotes and Leu carriers, respectively) were recruited from our initial cohort for participation in the fMRI study after exclusion of contraindications. Three of these participants had to be excluded due to technical problems, leaving 67 participants for data analysis (see Table 4 for detailed demographics). All participants gave written informed consent in accordance with the Declaration of Helsinki and received financial compensation for participation. The work was approved by the Ethics Committee of the University of Magdeburg, Faculty of Medicine.

\section{GENOTYPING}

Genomic DNA was extracted from blood leukocytes using the GenElute DNA extraction kit (Sigma Aldrich) according to the manufacturer's protocol. Genotyping was performed using PCR followed by allele-specific restriction analysis. The DNA fragment on Chr 14q21-24 containing the AKAP5 Pro100Leu polymorphism was amplified using the primers AKAP5_100- $f\left(5^{\prime}-\mathrm{GCT}\right.$ TCT GAT CAG CCA GAG CCC AC-3') and AKAP5_100-r $\left(5^{\prime}-\right.$ GCT TCT TCC TGG ACT TTG ATG CTG CAG-3') and standard Taq polymerase (Qiagen and Fermentas). PCR products were digested with $A l u \mathrm{I}$ (Fermentas), yielding two fragments $(174+98 \mathrm{bp})$ for the Leu allele or a single fragment (272 bp) for 
the Pro allele. DNA fragments were separated on an ethidium bromide-stained agarose gel and visualized under UV light (see Figure 1C for genotyping example). All subjects suspected to be homozygous for the less common Leu allele were genotyped twice. The rater was blinded to the results of the personality inventories. PCR products from two homozygous subjects (one $\mathrm{P} / \mathrm{P}$, one $\mathrm{L} / \mathrm{L}$ ) were verified via custom sequencing (SeqLab), confirming that the PCR reaction had provided the desired DNA fragment on Chr $14 \mathrm{q} 21-24$.

To avoid population stratification effects and to exclude asymmetric distribution of other genetic variations that might affect aggression or anger, participants were also genotyped for the BDNF Val66Met polymorphism (Chr 11p13), the COMT Val108/158Met polymorphism (Chr 22q11), the DRD2 TaqIA polymorphism (Chr 11q23), the DRD3 Ser9Gly polymorphism (Chr 3q13), the MAOA VNTR polymorphism (Chr Xp11), the serotonin transporter fragment length polymorphism (Chr 17q11-12), for the serotonin receptor 5HT-2a His452Tyr polymorphism (Chr 13q14-21), and for additional polymorphisms in pre- and post-synaptic adaptor proteins (detailed genotyping protocols are available upon request), and $X^{2}$ tests were used to compare the genotype distributions of these polymorphisms between AKAP5 Pro homozygotes and Leu carriers.

\section{QUESTIONNAIRE STUDY}

\section{Personality questionnaires}

Participants completed two well-established questionnaires that assess interindividual variability of aggression and anger. Selfrating on aggressive behavior was assessed using the German version of the BPAQ. The 29-item questionnaire (Buss and Perry, 1992) assesses four factors of aggressive behavior: physical aggression, verbal aggression, anger, and hostility.

The self-rating concerning the emotional state of anger was surveyed with the state-trait anger expression inventory (STAXI) developed by Spielberger (1991); German version by Schwenkmezger and Hodapp, 1991). The STAXI contains five anger-related subscales: the current level of anger (state anger), anger as personality trait (tendency to experience anger, i.e., trait anger); Anger-out behavior (AO; frequency with which anger is expressed toward other people or objects); Anger-in behavior (AI; directing anger toward oneself or inwardly; also related to suppressed hostility); Anger-control behavior (AC; active management of feeling angry in order to avoid anger expression).

\section{Statistical analysis}

To examine the overall influence of AKAP5 on measures of aggressive behavior and anger expression, we investigated the relationship between AKAP5 genotype and the four domains of the BPAQ (physical aggression, verbal aggression, anger, hostility) as well as four out of five domains of the STAXI (trait anger, anger-in, anger-out, anger control) as dependent variables. The state anger dimension of the STAXI was not included, as this dimension is defined as a temporary emotional state and no stable quality of the person. Therefore, possible genetic influences on the state measure would most likely be disguised by considerable intra-individual variability at any given time of testing. To circumvent the problem of multiple testing, we computed a single multivariate analysis of variance (MANOVA) with the above mentioned test scores as dependent variable and AKAP5 Pro100Leu (Pro homozygotes vs. Pro/Leu and Leu/Leu) as independent variable of interest. Because of the unequal distribution of the polymorphism in males and females in our cohort (see below) and previous reports showing higher aggression in males, we included gender as a further independent variable. Age, which was slightly lower in Leu carriers (see below), was included as a covariate of no interest. To ensure that all data met the required assumptions for MANOVA, we ensured that the sample size was large $(N>50$ for all cells), and we computed Levene's test of variance homogeneity for all dependent variables. Because variance homogeneity was not met for the dimension of anger control $\left(F_{3}=5.858 ; p=0.001\right)$, we adjusted the significance level for all post hoc tests to.025, as previously suggested for this case (Tabachnick and Fidell, 1983).

Subscores with significant effects of AKAP5 genotype in the MANOVA were verified using one-tailed post hoc two-sample $t$ tests, using a Bonferroni correction for multiple comparisons. To further verify a possible specific influence of AKAP5 Pro100Leu on certain BPAQ and STAXI subscores, a linear discriminant analysis (LDA) was performed, into which all eight subscores were entered in a stepwise fashion.

\section{FUNCTIONAL MRI EXPERIMENT Paradigm}

Emotional stimuli have previously been demonstrated to interfere with attentional processing, among others in the Eriksen flanker task (Fenske and Eastwood, 2003; Larson et al., 2006; Wiswede et al., 2009). For instance, Wiswede et al. (2009) could demonstrate that the presentation of unpleasant pictures from the International Affective Picture System (IAPS) prior to each flanker stimulus led to an increased ERN compared to trials with neutral or pleasant IAPS pictures.

The aim of the present study was to investigate the neural mechanisms underlying the cognitive control of anger-related emotional interference. Van Honk et al. (2001) had previously demonstrated that attentional biases for angry faces are related to trait anger. In relation to that observation and to our behavioral finding of Leu carriers showing a higher anger control, we hypothesized a genetically mediated performance advantage for Leu carriers in an emotional flanker task. Flanker stimuli, comprising a central target arrow pointing to the left or right side and requiring a button press with the corresponding left or right index finger were flanked by irrelevant arrows either pointing in the same (=congruent trials) or opposite (=incongruent trials) direction. Flanker stimuli were superimposed on neutral or emotional pictures (Van Honk et al., 2001; Larson et al., 2006; Wiswede et al., 2009). According to the concept of embodying emotion (Niedenthal, 2007) it is assumed that the perception of emotional expressions evokes a re-experiencing of the specific emotion in one's self. Because we were particularly interested in anger control processing, faces with either neutral or angry expressions were chosen as background stimuli. Face stimuli were obtained from the Karolinska Directed Emotional Faces database (KDEF; Lundqvist et al., 1998) and converted into black and white.

Figure 3A depicts the structure of an example trial. All trials started with the presentation of a face (neutral or angry) for 
$650 \mathrm{~ms}$, followed by a flanker stimulus (congruent or incongruent) for $200 \mathrm{~ms}$, during which the face stimulus was blurred, and the presentation of the face stimulus for another $650 \mathrm{~ms}$. The intertrial interval was jittered near-exponentially between 2 and $8 \mathrm{~s}$, to optimize estimation of the trial-specific hemodynamic response functions (HRFs; Hinrichs et al., 2000). To further improve our estimation concerning the trial-specific HRFs, we included a baseline condition in which the target stimulus was flanked by neutral stimuli ("_") and presented with a blurred background face. The experiment consisted of two runs, both comprising of 40 trials of each condition (high vs. low interference $\times$ emotional vs. neutral; baseline).

\section{Image acquisition}

Functional magnetic resonance imaging was performed using a GE Signa 1.5 T magnetic resonance system (General Electric) and a standard head coil. Two sessions of 428 echo-planar images (EPIs) were acquired in an interleaved manner (23 axial slices; voxel size $=3.13 \mathrm{~mm} \times 3.13 \mathrm{~mm} \times 4 \mathrm{~mm}+1 \mathrm{~mm}$ gap; $\mathrm{TR}=2 \mathrm{~s}$; $\mathrm{TE}=35 \mathrm{~ms}$; odd numbers first). Additionally, a co-planar protondensity (PD)-weighted MR image was acquired and used for coregistration to improve spatial normalization.

\section{Data processing and analysis}

Statistical analyses were performed using Statistical Parametric Mapping (SPM8, Wellcome Department of Imaging Neuroscience, London, UK). EPIs were corrected for acquisition delay and head motion. The co-planar PD-weighted image was coregistered to the mean image obtained from motion correction and used as reference image for spatial normalization to the Montreal Neurological Institute $(\mathrm{MNI})$ stereotactic coordinate system (voxel size $=3 \mathrm{~mm} \times 3 \mathrm{~mm} \times 3 \mathrm{~mm}$ ), the co-planar PD-weighted image was used to determine normalization parameters.

Statistical analysis was carried out using a two-stage mixed effects model. In the first stage, the hemodynamic response was modeled by convolving a delta function at stimulus onset with a canonical HRF (Friston et al., 1998). The resulting time courses were down sampled for each scan to form covariates of a general linear model (GLM). The model included separate covariates for each condition of interest (emotional incongruent, neutral incongruent, emotional congruent, neutral congruent, baseline), the six rigid-body movement parameters determined from realignment as covariates of no interest, and a single constant representing the mean over scans. Model estimation was performed using a restricted maximum likelihood fit, and contrasts of parameter estimates were computed for the comparisons of interest (emotional incongruent $>$ neutral incongruent and emotional congruent $>$ neutral congruent).

In the second stage of the model, the conditions of interest (emotional vs. neutral in the incongruent and congruent condition, separated by genotype) were submitted to second level random effects analyses. Specifically, planned $t$-test comparisons were computed for the emotional interference contrasts in the incongruent and congruent conditions, respectively, and masked inclusively with the genotype by condition interaction $F$ contrast (thresholded at $p<0.05$ ). Because of our strong a priori hypotheses derived from the questionnaire data that AKAP5 genotype would affect emotional interference processing in the anterior cingulate (and adjacent dorsomedial PFC) and possibly in the OFC, we focused our analyzes on ACC and OFC ROIs. ROIs were defined by Brodmann Areas (BAs 24, 32, and 6 for the ACC ROIs; BA 11 for the OFC ROI) and extracted from the WFU PickAtlas (Wake Forest University), and the significance level was set to $p<0.05$, ROI-volume-corrected for family wise error (FWE). Peak activation differences (SPM contrasts of parameter estimates) within these ROIs were submitted to confidence interval estimation using Bootstrap resampling and the percentile- $t$ method (Efron and Tibshirani, 1993; Schott et al., 2006). Only activation differences that showed non-overlapping confidence intervals for Pro homozygotes and Leu carriers were considered reliable.

To test whether genetically mediated ACC activation differences were associated with performance differences on the behavioral level, we computed a stepwise linear regression analysis with ACC activation in the high interference condition (emotional vs. neutral incongruent) as dependent variable and the RTs in the four conditions (incongruent vs. congruent $\times$ emotional vs. neutral) as independent variables.

\section{IMMUNOHISTOCHEMISTRY AND WESTERN BLOTTING Human brain material}

Brain material was obtained from the New Magdeburg Collection (Department of Psychiatry and Psychotherapy, University Hospital Magdeburg, Germany). The collection of human brain material has been carried out in accordance with German laws and the rules outlined by the local ethics committee. Brains from four individuals (one 50-year-old female and three males of age 48,54 , and 56 years) without any signs of neurological or psychiatric disorders were studied. The postmortem intervals ranged from 11 to $24 \mathrm{~h}$. Two brains were flash-frozen in liquid nitrogen for biochemical purposes, and the other two were processed for immunohistochemistry.

\section{Immunohistochemical procedures}

Brain tissue was processed for histochemical analyses in a standard manner, including immersion fixation in $8 \%$ phosphate-buffered formaldehyde for 2 months, embedding in paraplast, and cutting with a microtome (whole brain sections, $20 \mu \mathrm{m}$ ). Every $50^{\text {th }}$ section was histologically stained by combining the methods of Nissl and Heidenhain-Woelcke. To localize AKAP5 immunoreactivity, we used a commercially available mouse monoclonal antibody raised against the human AKAP79 protein (Santa Cruz Biotechnology, Santa Cruz, CA, USA). The working dilution of the primary antiserum was 1:100 in PBS. The nickel-enhanced avidin-biotin technique was used as described previously (Bernstein et al., 1999). The specificity of immunoreactivity was controlled by replacing the antibody with buffer and Ig-free serum. Brain regions were selected for AKAP staining based on the results of our fMRI study.

\section{Western blotting}

Protein preparation and Western blotting were essentially performed as described previously (Seidenbecher et al., 2002; Schott et al., 2006), with minor modifications. Briefly, human brain tissue from the hippocampus (including dentate gyrus and CA1 and CA3 
regions), the cingulate cortex and from the cerebellum was homogenized in $20 \mathrm{mM}$ HEPES ( $\mathrm{pH} 7.4$ ) containing $20 \mathrm{mM} \mathrm{NaCl}, 5 \mathrm{mM}$ EDTA, and $1 \%$ Triton X-100 and centrifuged at $20.000 \times g$ for $30 \mathrm{~min}$. Protein probes from the pellet and supernatant were solubilized with SDS and mercaptoethanol. Proteins were separated by SDS-PAGE on 5-20\% gels under fully reducing conditions, and transfer onto nitrocellulose was performed according to standard protocols. Western blots were incubated overnight with the primary antibody also used for immunohistochemistry and developed using the enhanced chemiluminescence detection system (Amersham, Arlington Heights, IL, USA).

\section{RESULTS}

\section{EFFECTS OF AKAP5 PRO100LEU ON HUMAN AGGRESSION AND ANGER}

To assess a potential influence of AKAP5 Pro100Leu on human aggression and anger, we systematically investigated genotypedependent group differences in well-established self-report questionnaires of aggressive behavior and anger expression in a cohort recruited from the interdisciplinary campus community of the University of Magdeburg, Germany. From 527 young, healthy participants (289 women, 238 men), complete genetic, and questionnaire data were available. In this cohort, we identified 370 Pro homozygotes, 150 heterozygotes, and 7 Leu homozygotes. With $15.6 \%$ frequency of the Leu allele and 28\% heterozygosity, the distribution was at Hardy-Weinberg equilibrium $\left(X^{2}=3.646\right.$; $p=0.162$ ), and frequencies were similar to those observed previously in a Caucasian population (Frank et al., 2008). Detailed demographics are shown in Table 1. To avoid population stratification effects, participants were also genotyped for additional polymorphisms in unrelated genes (see Materials and Methods). The polymorphisms were observed at allele frequencies similar to those reported previously, and no imbalance was observed in the distribution of any of these polymorphisms between Pro homozygotes and Leu carriers (all $p>0.133$ ). However, the distribution varied between male and female participants, with the Leu allele being significantly more prevalent in females $\left(X^{2}=9.37\right.$; $p=0.009$; Figure 1D; see supplemental information for discussion). Given the low number of Leu homozygous subjects $(n=7)$, all Leu carriers (Pro/Leu and Leu/Leu) were grouped together for all further analyzes.

Buss-Perry aggression questionnaire and STAXI results are displayed in Figure 2 (see also Table 2), separated by AKAP5 genotype. Because of the unequal distribution of AKAP5 Pro100Leu across gender and because of a small age difference between genotypes (Table 1), we computed a MANOVA with gender and AKAP5 genotype as independent factors and age as covariate. Dependent variables were the four dimensions of the BPAQ and all STAXI domains except for state

Table 1 | Demographic data of the behavioral study.

\begin{tabular}{llll}
\hline & Pro/Pro & Pro/Leu, Leu/Leu & \\
\hline W/M & $191 / 179$ & $98 / 59$ & $X^{2}=9.37 ; p=0.009^{*}$ \\
Age & $23.1 \pm 3.1$ & $22.4 \pm 2.9$ & $t=2.19 ; p=0.029^{*}$
\end{tabular}

W/M, women/men. Mean age and SD are shown.

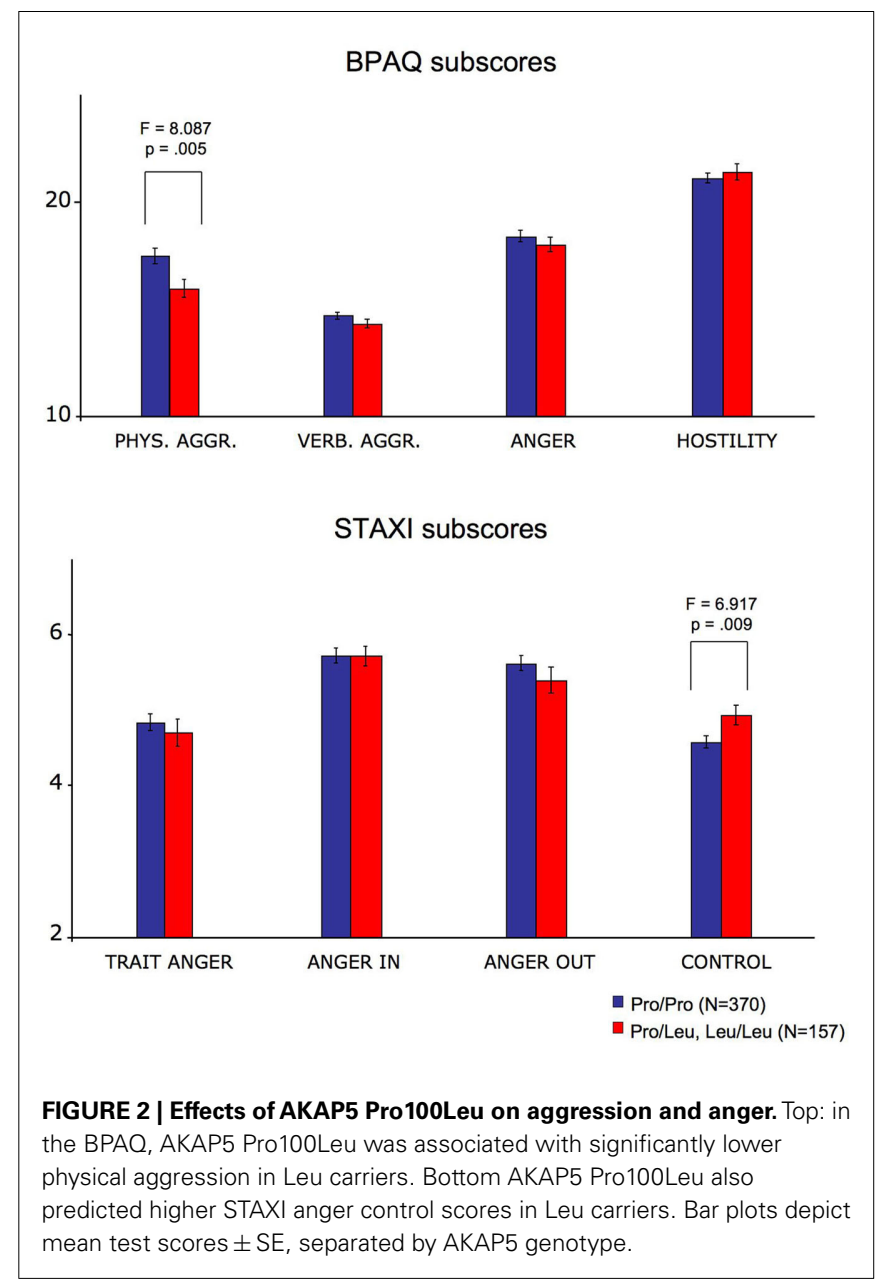

anger. The MANOVA yielded main effects of AKAP5 genotype and gender, but no significant interaction (Table 2, bottom). There was also a significant main effect of age (see below). Between-subjects univariate ANOVAs yielded significant effects of AKAP5 genotype on the physical aggression scale of the BPAQ $\left(F_{1,522}=8.087 ; p=0.005 ; \eta^{2}=0.015\right)$ and on the anger control scale of the STAXI $\left(F_{1,522}=6.917 ; p=0.009 ; \eta^{2}=0.013\right)$. Post hoc one-tailed two-sample $t$-tests showed that AKAP5 Leu carriers had significantly lower scores in physical aggression $\left(t_{525}=2.874 ; p=0.004\right.$, Bonferroni-corrected) and significantly higher scores in anger control $\left(t_{530}=2.354 ; p=0.019\right.$, Bonferroni-corrected).

To test, which of the dependent variables distinguished best between AKAP5 Pro homozygotes and Leu carriers, the four domains of interest from the BPAQ (physical aggression, verbal aggression, anger, hostility) and the STAXI scales for trait anger, anger-in, anger-out, and anger control were entered into a stepwise LDA. The LDA showed that physical aggression (Wilk's lambda $\left.=0.985 ; F_{1,1,525}=8.262 ; p=0.004\right)$ and anger control (Wilk's lambda $=0.975 ; F_{2,1,524}=6.687 ; p=0.001$ ) separated the two genotype groups best, with no other subscores of either questionnaire entering the model.

In order to validate our results obtained in the German cohort we performed post hoc genotyping for AKAP5 Pro100Leu 
in a previously described cohort of 604 participants from the University of Barcelona community (457 female, mean age $21.6 \pm 3.1$ years; see Krämer et al., 2007, for detailed demographics). The participants had completed the Spanish version of the BPAQ, but no STAXI scores data were available from that cohort. Table 3 displays demographic data and BPAQ scores. Carriers of the Leu allele showed trends for lower total BPAQ scores $(t=1.515 ; p=0.065$, one-tailed $)$ as well as for the domains physical aggression $(t=1.478 ; p=0.073$, one-tailed), hostility ( $t=1.666 ; p=0.048$, one-tailed), and, to a lesser degree, anger $(t=1.128, p=0.130$, one-tailed). These trends could be observed across the whole group and in female participants alone.

\section{GENDER AND AGE EFFECTS ON BPAO AND STAXI SCORES}

While age and gender did affect BPAQ and STAXI scores, they exerted their influence on domains distinct from those affected by AKAP5 Pro100Leu genotype. In our MANOVA model, a significant gender effect was observed for the anger-out dimension of the STAXI $\left(F_{1,522}=4.292 ; p=0.039 ; \eta^{2}=0.008\right)$, but not for either physical aggression or anger control (all $p>0.523$ ). At the level of between-subject comparisons, we also observed a significant genotype by gender interaction for anger control $\left(F_{1,522}=6.740\right.$; $\left.p=0.010 ; \eta^{2}=0.013\right)$. Age exerted a significant effect on the trait anger dimension of the STAXI $\left(F_{1,529}=10.051 ; p=0.002\right.$; $\left.\eta^{2}=0.019\right)$, but there were no age effects on either physical or verbal aggression or on anger control (all $p>0.131$ ). Post hoc Pearson correlations showed a weak but significant negative correlation between age and trait anger $(r=-0.121 ; p=0.005)$ and a trend for a negative correlation between age and the anger-out scale $(r=-0.084 ; p=0.053)$. No further significant correlations between age and BPAQ or STAXI subscores were observed (all $p>0.203)$.

\section{EFFECTS OF AKAP5 PR0100LEU ON NEURAL CORRELATES OF EMOTIONAL INTERFERENCE}

To further explore the neural mechanisms that might underlie the observed associations of AKAP5 Pro100Leu with aggression and anger control, we conducted an fMRI study in 67 young, healthy participants (34 Pro homozygotes, 33 Leu carriers). Because of the observed effect of AKAP5 Pro100Leu on anger control and the known trait anger-related attentional bias for angry faces (Van Honk et al., 2001), we hypothesized that Pro homozygotes and Leu carriers might differ at neural level during the cognitive control of emotional interference. We addressed this hypothesis by using an Erikson flanker paradigm with task-irrelevant background pictures (Larson et al., 2006; Wiswede et al., 2009), which, in our study, were angry vs. neutral faces (Figure 3A). In the Eriksen flanker paradigm, processing of incongruent stimuli is typically associated with activations in medial frontal cortex ( $\mathrm{mFC}$ ) regions like the supplementary motor area (SMA, Brodmann area/BA 6) and the ACC (Ridderinkhof et al., 2004).

Table 3 | Demographic data and mean BPAQ scores of Spanish cohort.

\begin{tabular}{lll}
\hline & Pro/Pro & Pro/Leu, Leu/Leu \\
\hline W/M & $316 / 109$ & $141 / 38$ \\
Mean age & $21.7 \pm 3.2$ & $21.3 \pm 2.8$ \\
Total BPAO & $65.1 \pm 15.4$ & $63.2 \pm 13.1$ \\
Physical aggression & $15.5 \pm 5.3$ & $14.9 \pm 4.8$ \\
Verbal aggression & $13.1 \pm 3.9$ & $13.1 \pm 3.3$ \\
Anger & $17.5 \pm 5.4$ & $17.1 \pm 4.7$ \\
Hostility & $18.9 \pm 5.5$ & $18.1 \pm 4.9$
\end{tabular}

W/M, women/men.

Table 2 | Effects of AKAP5 Pro100Leu on BPAQ and STAXI scores.

\begin{tabular}{|c|c|c|c|c|c|c|c|c|}
\hline & \multicolumn{4}{|c|}{ BPAQ } & \multicolumn{4}{|c|}{ STAXI } \\
\hline & Physical aggression & Verbal aggression & Anger & Hostility & Trait anger & Anger-in & Anger-out & Anger control \\
\hline \multicolumn{9}{|l|}{ Pro } \\
\hline W & $17.6 \pm 5.84$ & $14.7 \pm 2.71$ & $18.7 \pm 4.67$ & $21.2 \pm 4.60$ & $4.8 \pm 2.23$ & $5.8 \pm 1.62$ & $5.4 \pm 1.99$ & $4.8 \pm 1.41$ \\
\hline M & $17.3 \pm 5.94$ & $14.6 \pm 2.60$ & $18.0 \pm 4.70$ & $21.0 \pm 4.27$ & $5.0 \pm 2.29$ & $5.6 \pm 1.87$ & $5.8 \pm 1.88$ & $4.3 \pm 1.77$ \\
\hline \multicolumn{9}{|l|}{ Leu } \\
\hline W & $15.8 \pm 5.16$ & $14.3 \pm 2.65$ & $18.3 \pm 4.15$ & $21.1 \pm 5.18$ & $4.6 \pm 2.31$ & $5.6 \pm 1.81$ & $5.3 \pm 2.15$ & $4.8 \pm 1.70$ \\
\hline \multirow[t]{2}{*}{$M$} & $16.1 \pm 4.95$ & $14.3 \pm 2.80$ & $17.4 \pm 4.28$ & $21.8 \pm 4.25$ & $4.9 \pm 2.34$ & $5.9 \pm 1.73$ & $5.5 \pm 2.05$ & $5.1 \pm 1.56$ \\
\hline & \multicolumn{2}{|c|}{ AKAP5 } & \multicolumn{2}{|c|}{ GENDER } & \multicolumn{2}{|c|}{ AKAP5 $\times$ GENDER } & \multicolumn{2}{|c|}{ AGE } \\
\hline Wilk's $\lambda$ & \multicolumn{2}{|c|}{0.966} & \multicolumn{2}{|c|}{0.960} & \multicolumn{2}{|c|}{0.983} & \multicolumn{2}{|c|}{0.970} \\
\hline$F_{8,515}$ & \multicolumn{2}{|c|}{2.260} & \multicolumn{2}{|c|}{2.653} & \multicolumn{2}{|c|}{1.093} & \multicolumn{2}{|c|}{2.022} \\
\hline$p$ & \multicolumn{2}{|c|}{$0.022^{*}$} & \multicolumn{2}{|c|}{$0.007^{*}$} & \multicolumn{2}{|c|}{0.367} & \multicolumn{2}{|c|}{$0.042^{*}$} \\
\hline$\eta^{2}$ & \multicolumn{2}{|c|}{0.034} & \multicolumn{2}{|c|}{0.030} & \multicolumn{2}{|c|}{0.017} & \multicolumn{2}{|c|}{0.034} \\
\hline
\end{tabular}

Top: test scores separated by gender and AKAP5 genotype (means and SD are shown). BPAQ, Buss-Perry aggression questionnaire; STAXI, state-trait anger expression inventory; Pro, 100Proline carriers; Leu, 100Leucine carriers; W, women; M, men. Bottom: results of the MANOVA (fixed factors: AKAP5; gender; covariate: age), ${ }^{*} p<0.05$. 
A

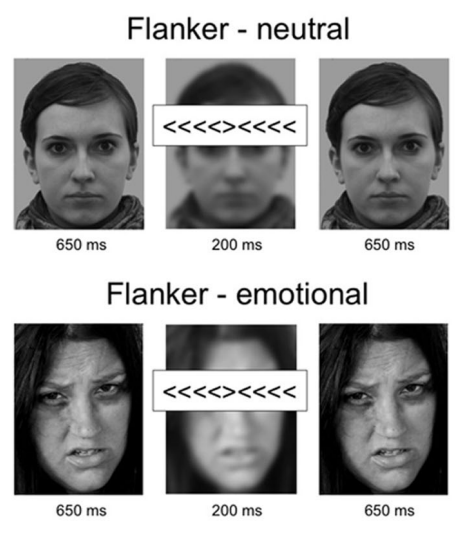

B

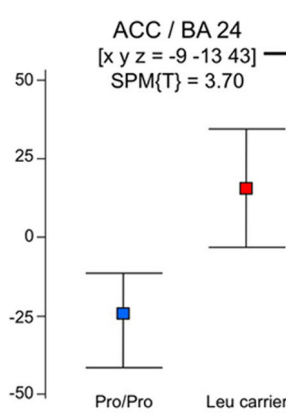

FIGURE 3 | Functional MRI correlates of emotional interference. (A) Schematic illustration of the experimental paradigm in Section "Materials and Methods" for details. (Note: the example face stimuli in this figure and Figure $\mathbf{4}$ are not part of the actual stimulus set used in the experiment, but were created by the authors for illustrative purposes.) (B) Leu carriers exhibited increased activation of the ACC (BA 24, extending into BA 6) for emotional vs. neutral background pictures in the

\section{Behavioral results}

Demographic and behavioral data of the fMRI study are summarized in Table 4. Error rates were significantly higher in the incongruent relative to the congruent flanker condition (main effect of congruency: $F_{1,65}=42.56$; $p<0.001$; two-way ANOVA for repeated measures with AKAP5 genotype as between-subjects factor). There were also slightly higher error rates in the emotional vs. neutral conditions $\left(F_{1,65}=4.33 ; p=0.041\right)$, but no significant interaction between congruency and emotional vs. neutral background pictures $\left(F_{1,65}=2.42 ; p=0.125\right)$. Post hoc one-tailed $t$-tests suggested that the effects of emotionality on error rates were mainly driven by the incongruent condition (incongruent: $T_{66}=1.892$; $p=0.032$; congruent: $T_{66}=1.097 ; p=0.139$ ). AKAP5 genotype did not influence error rates in any condition (all $p>0.314)$.

Reaction times were significantly longer in the incongruent relative to the congruent condition $\left(F_{1,65}=226.22 ; p<0.001\right.$; two-way ANOVA for repeated measures with AKAP5 genotype as between-subjects factor), but there was no effect of emotional background on RTs and no interaction between congruency and emotion (all $p>0.488$ ). A nominal tendency for shorter RTs in Leu carriers relative to Pro homozygotes in all conditions was not significant (main effect of AKAP5 and all interactions with AKAP5 genotype: all $p>0.246)$.

\section{Functional MRI results}

$T$-test based ROI analyses comparing the emotional vs. neutral contrasts in the incongruent condition revealed that AKAP5 Leu carriers showed a significantly stronger emotion-dependent activation of a cluster within the left (ACC, BA 24, extending into the supplementary motor area, SMA, BA 6; $p=0.019$, FWE-corrected for ROI volume) when compared to Pro homozygotes (Figure 3B). This activation difference showed non-overlapping 95\% confidence intervals for Pro homozygotes and Leu carriers as estimated using Bootstrap resampling and the percentile- $t$ method (Efron and Tibshirani, 1993; Schott et al., 2006). We also observed a tendency for increased emotion-dependent activation of the right ACC [BA 32; $(x y z)=(123528)]$ in Leu carriers during incongruent trials, but this activation difference showed only a trend after small-volume FWE correction ( $p=0.071$, FWE-corrected for ROI volume).

Pro homozygotes, on the other hand showed relatively increased activity in the right medial OFC during incongruent emotional trials when compared to Leu carriers. The activation difference in the OFC was significant after small-volume FWE correction ( $p=0.003$, FWE-corrected for ROI volume), and Bootstrap-based $95 \%$ confidence intervals did not overlap between Pro homozygotes and Leu carriers.

In the congruent condition, the pattern observed in the incongruent condition was partly reversed. Here, Pro homozygotes 
Table 4 | Demographic and behavioral data of the fMRI experiment.

\begin{tabular}{|c|c|c|c|c|c|}
\hline & \multicolumn{2}{|r|}{ Pro/Pro } & \multicolumn{2}{|c|}{ Pro/Leu, Leu/Leu } & \multirow[b]{3}{*}{$X^{2}=0.002 ; p=0.888$} \\
\hline & $W$ & $M$ & $W$ & $M$ & \\
\hline$N$ & 18 & 16 & 19 & 14 & \\
\hline Mean age & $24.1 \pm 2.9$ & $25.4 \pm 2.0$ & $23.9 \pm 2.0$ & $25.6 \pm 4.2$ & $F=0.01 ; p=0.972$ \\
\hline \multicolumn{6}{|c|}{ Error rates } \\
\hline \multicolumn{6}{|c|}{ Incongruent } \\
\hline Emotional & \multicolumn{2}{|r|}{$3.18+4.76$} & \multicolumn{2}{|c|}{$3.94 \pm 4.87$} & \\
\hline Neutral & \multicolumn{2}{|r|}{$2.85 \pm 3.95$} & \multicolumn{2}{|c|}{$3.18 \pm 3.85$} & \\
\hline \multicolumn{6}{|l|}{ Congruent } \\
\hline Emotional & \multicolumn{2}{|r|}{$0.26 \pm 0.83$} & \multicolumn{2}{|c|}{$0.27 \pm 0.63$} & \\
\hline Neutral & \multicolumn{2}{|r|}{$0.26 \pm 0.71$} & \multicolumn{2}{|c|}{$0.09 \pm 0.38$} & \\
\hline \multicolumn{6}{|l|}{ RTs (ms) } \\
\hline \multicolumn{6}{|c|}{ Incongruent } \\
\hline Emotional & \multicolumn{2}{|r|}{$594 \pm 106.7$} & \multicolumn{2}{|c|}{$570 \pm 111.6$} & \\
\hline Neutral & \multicolumn{2}{|r|}{$597 \pm 105.6$} & \multicolumn{2}{|c|}{$569 \pm 113.5$} & \\
\hline \multicolumn{6}{|l|}{ Congruent } \\
\hline Emotional & \multicolumn{2}{|r|}{$494 \pm 64.1$} & \multicolumn{2}{|c|}{$470 \pm 72.3$} & \\
\hline Neutral & \multicolumn{2}{|r|}{$494 \pm 63.4$} & \multicolumn{2}{|c|}{$471 \pm 73.0$} & \\
\hline
\end{tabular}

Mean error rates and reaction times $\pm S D$ are shown. $R T$, reaction time; $W / M$, women/men.

showed a stronger ACC activation relative to Leu carriers [BA24; $\left(\begin{array}{lll}x & y & z\end{array}\right)=(-15-449) ; p=0.048$, FWE-corrected for ROI volume], and this activation difference was robust after bootstrap resampling. No FWE-correctable genotype-related activation differences in either direction were observed in the OFC during the congruent condition.

\section{Brain-behavior correlations}

Across our study cohort, we observed a negative correlation of RTs with emotion-related ACC activation peak in the incongruent condition. A stepwise multiple regression analysis showed that this RT advantage was most prominent in the emotional incongruent condition itself $(\beta=-0.258 ; p=0.035)$. Neither OFC activation differences during the incongruent condition and the peak activation difference in the ACC during the congruent condition showed a correlation with RTs, and neither ACC nor OFC activations were significantly correlated with error rates in any condition (all $p>0.1$.

\section{EXPRESSION OF AKAP5 IN HUMAN FRONTOLIMBIC CORTICES}

Previous studies had demonstrated AKAP5 expression in the human amygdala, hippocampus and striatum, but no information was thus far available on the expression of AKAP5 in frontolimbic cortical structures. In our experiments AKAP5 Pro100Leu affected cortical rather than subcortical limbic activity during emotional interference. Therefore, expression of AKAP5 in the human frontal limbic neocortex was taken into account. We used immunohistochemistry on post mortem human brain tissue to assess expression of AKAP5 in human anterior cingulate, medial frontal, and orbitofrontal regions, based on our fMRI results. Indeed, we found AKAP5 immunoreactivity widely distributed in the human cortex. In the ACC (BA 24, 32) and in the mFC (BA 6) AKAP5 was expressed in pyramidal cells and a subset of interneurons (Figure 4A, top row, middle row, left). A similar expression pattern was observed in the medial OFC (BA 11). To verify the validity of these findings, AKAP5 immunoreactivity was also assessed in the hippocampus and striatum, brain regions that have previously been shown to express AKAP5 (Sik et al., 2000; Ulfig et al., 2001; Figure 4A, bottom row). Western blotting using the same primary antibody revealed expression of the previously observed AKAP5-immunoreactive band at approximately $75 \mathrm{kDa}$ (Gardner et al., 2006) in the detergent-insoluble pellet fractions of the human hippocampus and cingulate cortex (Figure 4B), but not in the cerebellum, compatible with previous observations for the AKAP5 ortholog in rodents (Ostroveanu et al., 2007), thus confirming the specificity of the antibody.

\section{DISCUSSION}

In our study cohort of 527 young, healthy participants, the AKAP5 Pro100Leu polymorphism was associated with human aggression and anger traits. Specifically, carriers of the less common Leu allele showed lower physical aggression and higher anger control relative to Pro homozygotes. In line with the behavioral data, Leu carriers exhibited stronger activations in the left ACC (BA 24) in our fMRI study of emotional interference. Complementary, Pro homozygotes showed a stronger activation in the right medial OFC during the same experimental condition.

\section{AKAP5 AND THE GENETIC VARIABILITY OF AGGRESSION AND ANGER}

Previous studies on genetically mediated variability of aggression and anger have largely focused on genes directly related to neuromodulatory, particularly monoaminergic, transmitter systems (Buckholtz and Meyer-Lindenberg, 2008; Kang et al., 2008; Kulikova et al., 2008; Hess et al., 2009). Here we could demonstrate that a genetic variation in an adaptor molecule that is involved most likely in intracellular signaling pathways of several monoaminergic neurotransmitter systems is associated with 


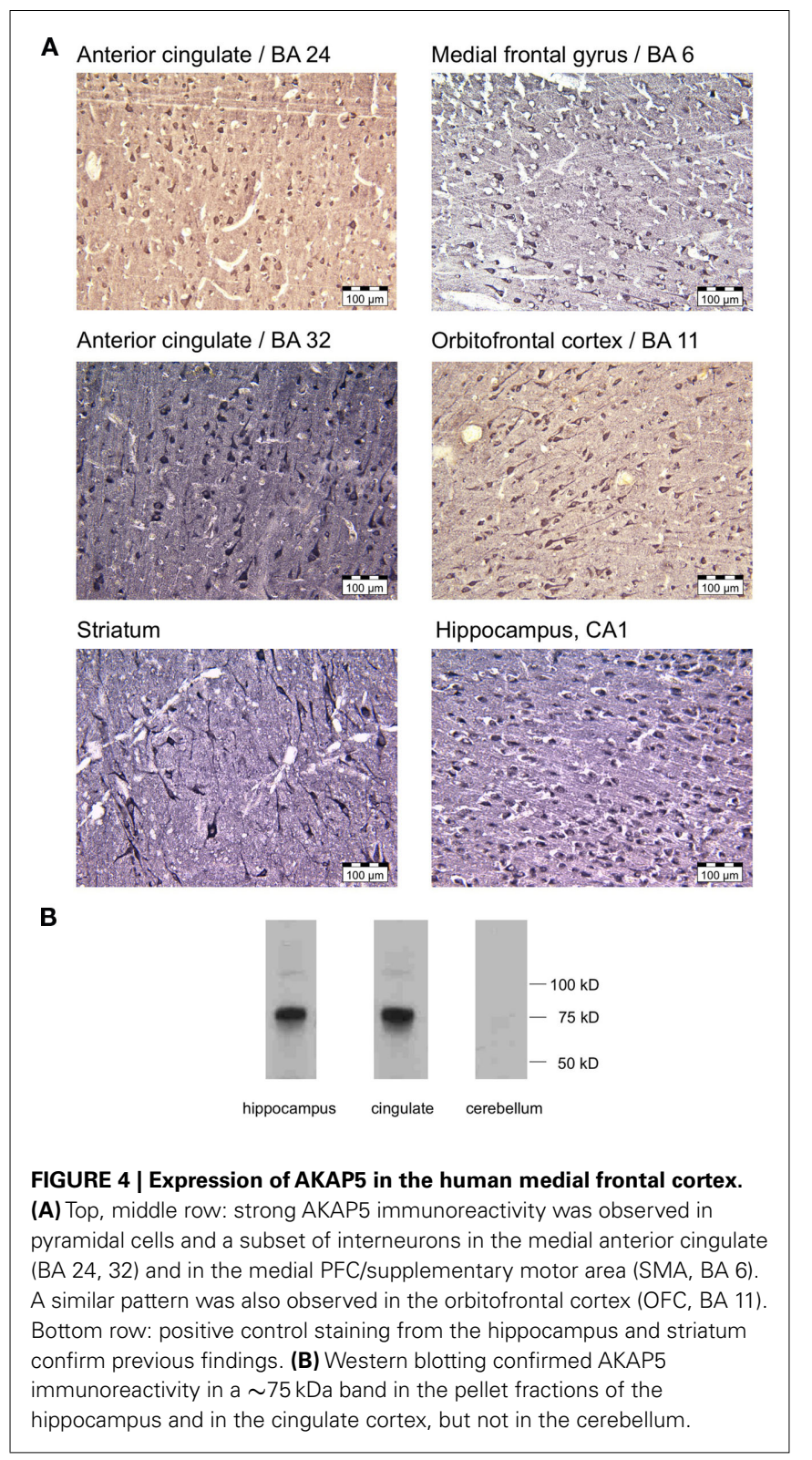

human anger and aggression phenotypes in young, healthy, participants. Notably, carriers of the less common variant show lower aggression and stronger anger control when compared to homozygotes of the presumed wild type allele. The association was observed in young, healthy participants and is therefore likely to reflect variability in the normal population. It should be noted that effect sizes were small, but given the complexity of both the phenotypes and the multiple other genetic and environmental influences, no larger influence could have been reasonably expected. In our analysis, effects of AKAP5 Pro100Leu were primarily observed for the dimensions of physical aggression and anger control, while other BPAQ and STAXI subscales showed trends into the same direction that did not reach significance.

The AKAP5 gene product interacts with beta-adrenergic receptors (Gardner et al., 2006), which are highly expressed in the mammalian brain. As beta-adrenergic signaling has been associated with human aggression (Fava, 1997; Hess et al., 2009), modulation of CNS beta-adrenergic activity might be a promising explanation for our observations. AKAP5 has also been linked to dopaminergic signaling and its interaction with glutamatergic neurotransmission (Swayze et al., 2004). Among the neuromodulatory monoamines, serotonin has previously been particularly strongly linked to aggressive behavior (Buckholtz and MeyerLindenberg, 2008; Popova, 2008). The role of AKAP5 in serotonergic signaling is thus far unclear, however, an in vitro study suggests a molecular association of AKAP150 with different $5 \mathrm{HT}$ receptors (Sandoz et al., 2006). Investigating a potential role of AKAP5 in the serotonergic system is therefore warranted to help explain the present results.

Because our study cohort consisted mainly of students, one might argue that it was somewhat stratified. It should be noted, though, that students were from a large variety of fields of studies (including economics, educational and natural sciences, and medicine, among others), and that the distribution of numerous additional genetic polymorphisms, which all occurred with the previously reported allele frequencies, showed no significant linkage disequilibrium with AKAP5 Pro100Leu. Moreover, the observation of a behavioral phenotype related to a genetic variation in a socially rather homogeneous population, such as the one investigated here, would be expected to be rather subtle and thus, if detected, even more likely to result from genetic differences rather than a sampling bias. Despite a small difference in the distribution of AKAP5 Pro100Leu between men and women (see Results), multivariate statistics revealed independent main effects of gender and genotype that were observed for distinct subscales.

Further though limited support for the validity of the present results comes from post hoc analysis of AKAP5 Pro100Leu on BPAQ scores in a previously reported cohort from Barcelona (Krämer et al., 2007), where comparable trends for lower aggression in Leu carriers were observed. Although the investigation of the AKAP polymorphism and its link to human aggression was not the primary goal in the original design of the Barcelona cohort, it yielded comparable trends for lower aggression in Leu carriers, but the effects were generally less pronounced and did not reach statistical significance in two-tailed comparisons. However, it should be noted that the participants from the Spanish cohort had overall lower BPAQ scores those from the German cohort. The most likely explanation for this might be that the majority of participants in the Spanish study were female (75.6\%), and women showed generally lower aggression. Furthermore, the lower overall BPAQ scores might also be related to linguistic or cultural aspects of the Spanish BPAQ version, as in an earlier validation study, García-León et al. (2002) had also reported lower scores in their Spanish subjects as compared Buss and Perry's original validation of the questionnaire in an American sample, and a direct comparison of BPAQ scores with Dutch and Japanese samples also revealed lower BPAQ scores in a Spanish cohort (Ramírez et al., 2001). The sensitivity of the BPAQ might also have been reduced to some degree by the fact that the participants from Barcelona were largely native speakers of Catalán rather than Spanish, but the Spanish version of the questionnaire was used. Support for the notion that the BPAQ might show linguistically or culturally mediated differences 
in sensitivity comes from several studies on adaptations of the BPAQ in different languages and cultures (Meesters et al., 1996; Nakano, 2001; Fossati et al., 2003). Those studies were able to confirm the four-factor structure, but reported disagreements on item level and concerning the interpretation of the factors (Von Collani and Werner, 2005), which might be reflected here by hostility being the BPAQ factor most strongly associated with AKAP5 Pro100Leu in the Spanish, but not in the German cohort. Future attempts to replicate our results in different ethnic and cultural populations should therefore include additional instruments, particularly the STAXI, which had not been obtained in the Barcelona cohort.

In the present study, we had focused our investigations on aggression and anger traits. It should be noted, however, that we cannot exclude further influences of the polymorphism on individual differences in emotional processing beyond aggression and anger. Previous animal research has demonstrated a role for AKAP5 in anxiety and fear memory (Moita et al., 2002; Nijholt et al., 2008; Tunquist et al., 2008). Further research is required to assess potential effects of AKAP5 Pro100Leu on anxiety and possibly on the processing of other negative emotions.

\section{AKAP5 PR0100LEU AND ITS EFFECT ON THE PROCESSING OF EMOTIONAL INTERFERENCE}

Compatible with significantly higher anger control in Leu carriers, our fMRI study showed stronger activation of the left (ACC, BA 24) in Leu carriers relative to Pro homozygotes when an angry face was presented in the background of an incongruent trial, that is, when emotional interference occurred in the condition that required higher attentional control. Increased activation of brain regions involved in attention and executive functions has previously been linked to decreased processing efficiency (Egan et al., 2001; Blasi et al., 2005; Meyer-Lindenberg and Weinberger, 2006). In those studies, increased prefrontal or ACC activation occurred despite similar or even inferior behavioral performance. Here we observed a significant correlation between the ACC BOLD signal in the emotional incongruent condition and shorter RTs. Moreover, as error rates did not significantly covary with the ACC response and were not higher in Leu carriers relative to Pro homozygotes, the reduced RTs likely reflect an actual behavioral advantage rather than impulsivity or a behavioral tradeoff, which would be associated with a corresponding error rate increase (Caldu et al., 2007). Compatible with our results, a recent study investigating the role of the well-described catechol-O-methyl transferase (COMT) Val108/158Met polymorphism on inhibitory control of memory has shown a similar pattern of increased cortical activity linked to better task performance. In that study, COMT Met carriers showed increased prefrontal activation during selective retrieval associated with more efficient inhibition of irrelevant memories (Wimber et al., 2011). Also, the observation that genetically mediated differences in brain activity predicted RTs more accurately than a direct comparison of the different allele carriers (see Results) is compatible with previous studies in which, similarly, fMRI correlates of cognitive processes showed correlations with both genetic variations and behavior that were stronger than the direct relationship between the respective genotypes and behavioral phenotypes (Bertolino et al., 2006; Forbes et al., 2009). For the commonly investigated COMT Val108/158Met polymorphism, a meta-analysis further demonstrated that BOLD responses are more sensitive to genetic modifiers as compared to direct behavioral measures, and the authors suggested that the fMRI endophenotype might reflect a neural mediator of pleiotropic effects of genetic variations on complex human behavior (Mier et al., 2010).

Given the observed relationship between increased ACC activation and shorter RTs, our results are in line with previously reported anger-related attentional biases for angry faces (Van Honk et al., 2001). While the ACC activation was found to be higher in Leu carriers, we observed a reverse effect in the right medial OFC, where Pro homozygotes exhibited a stronger fMRI response in the emotional incongruent condition, that is, in the same experimental condition where Leu carriers show their increased ACC activity. Moreover, activation of the ACC was observed in Pro homozygotes during emotional interference in the congruent condition. This activation might indeed reflect inefficient recruitment of the ACC as it was not correlated with a behavioral advantage during task performance.

Notably, these activation patterns reflect the previously proposed differentiation of a ventral affective processing system (VAPS) and a dorsal executive control system (DECS; Dolcos and Mccarthy, 2006). It is supposed that emotionally salient stimuli are processed in VAPS regions like the amygdala and the OFC while executive functions recruits DECS regions like the dorsolateral prefrontal cortex (DLPFC), posterior parietal cortex, and ACC (Pessoa, 2008; Hart et al., 2010). Related to our findings we suggest that Leu carriers, who exhibit higher anger control and a stronger ACC activation in the emotional incongruent flanker condition, emphasize the requested executive control during task performance. Pro homozygotes, in contrast, might react more sensitively to the emotional (angry) background stimuli, as reflected by a lower anger control and stronger activation of the VAPS in the emotional incongruent flanker condition.

Additionally, in order to test whether the observed effects of AKAP5 on emotional interference processing in the ACC and the OFC might reflect a local phenomenon related to monoaminergic modulation of ACC and OFC activity or rather the result of large-scale network activity, we had investigated the expression of AKAP5 in post mortem human brain tissue. Since AKAP5 was detected in both ACC and OFC pyramidal cells and interneurons (Figure 4), a locally mediated effect of AKAP5 on cognitive control of anger and aggression is possible, however, a more indirect mechanism, for example by influencing amygdala-dependent processing of anger, cannot be excluded. Although the mean age of the donors in the post mortem investigations ( 52.0 years) was substantially higher than that of the participants in the questionnaire and fMRI studies (22.9 years), it should be noted that mRNA profiling in younger donors ( 24 and 39 years, respectively) has also provided evidence for AKAP5 expression in frontolimbic structures (Allen's Brain Atlas ${ }^{1}$ ).

${ }^{1}$ http://human.brain-map.org 


\section{POTENTIAL MOLECULAR CONSEOUENCES OF AKAP5 PRO100LEU}

An important direction for future research will be the investigation of molecular consequences of AKAP5 Pro100Leu. The polymorphism is located close to the second basic membrane localization domain and the GPCR-binding region (Figure 1A) and affects the length of an alpha-helical structure and the geometry of the membrane-attached $\mathrm{N}$-terminus (Figure 1B), with a potential effect on the folding of the downstream domains (Protein folding was predicted using the PEP-FOLD prediction software ${ }^{2}$ ).

Given the localization of the polymorphism within the AKAP5 gene, we tentatively suggest that Pro100Leu might modulate interactions with GPCRs. The consequences of such an interaction are, however, be difficult to predict, particularly in relation to a complex behavioral phenotype such as aggression. AKAP5 Pro100Leu might, for example, affect the colocalization of GPCRs with their intracellular targets. Apart from a potential influence of AKAP5 interaction with GPCRs, it appears also plausible that the polymorphism might affect the interactions of AKAP5 with other post-synaptic adaptor molecules or with the post-synaptic membrane.

\section{CLINICAL IMPLICATIONS}

AKAP5 Pro100Leu has rarely been investigated thus far in clinical association studies, with only one published study in oncology, yielding a negative result (Frank et al., 2008). While AKAP5 Pro100Leu has not been investigated in the context of neurological or psychiatric disorders, a previous study suggested aberrant gene number variations of AKAP5 in schizophrenia and bipolar disorder (Wilson et al., 2006), although data are yet inconsistent (Sutrala et al., 2007). Given the high prevalence of aggressive behavior

${ }^{2}$ http://bioserv.rpbs.univ-paris-diderot.fr/PEP-FOLD/

\section{REFERENCES}

Berkowitz, L., and Harmon-Jones, E. (2004). Toward an understanding of the determinants of anger. Emotion 4, 107-130.

Bernstein, H. G., Baumann, B., Danos, P., Diekmann, S., Bogerts, B., Gundelfinger, E. D., and Braunewell, K. H. (1999). Regional and cellular distribution of neural visinin-like protein immunoreactivities (VILIP1 and VILIP-3) in human brain. $J$. Neurocytol. 28, 655-662.

Bertolino, A., Rubino, V., Sambataro, F., Blasi, G., Latorre, V., Fazio, L., Caforio, G., Petruzzella, V., Kolachana, B., Hariri, A., MeyerLindenberg, A., Nardini, M., Weinberger, D. R., and Scarabino, T. (2006). Prefrontal-hippocampal coupling during memory processing is modulated by COMT val158met genotype. Biol. Psychiatry 60, 1250-1258.

Blasi, G., Mattay, V. S., Bertolino, A., Elvevag, B., Callicott, J. H., Das, S., Kolachana, B. S., Egan, M. F., Goldberg, T. E., and Weinberger,

and impaired impulse control in psychiatric disorders, our results suggest AKAP5 an interesting candidate gene for future research on risk factors for neuropsychiatric diseases associated with aggression or impulsivity. We tentatively suggest that the Leu allele might confer a protective effect.

\section{CONCLUSION}

By using a multimodal approach including behavioral genetics, fMRI and post mortem immunohistochemistry, we could demonstrate that AKAP5 Pro100Leu is associated with human aggression and anger, with the Leu allele conferring a less aggressive phenotype with higher anger control. The fMRI results further suggest that individual differences in frontolimbic processing of emotional interference might constitute a neural basis of the higher anger control in Leu carriers. This is supported by immunohistochemical demonstration of AKAP5 expression in frontolimbic cortices. Our results indicate that future research on genetic mechanisms of aggression and anger should include intracellular signaling and adaptor proteins instead of only focusing on molecules directly involved in neuromodulatory transmission. Furthermore, our results suggest AKAP5 as a novel potential candidate gene for psychiatric disorders with altered aggression or impulse control.

\section{ACKNOWLEDGMENTS}

The authors would like to thank Gusalija Behnisch, Grazyna Debska-Vielhaber, and Maria Michelmann for help with genotyping. We further thank Helena Erlbeck, Kerstin Möhring, and Ilona Wiedenhöft for assistance with MRI acquisition and Estela Camara, Toni Cunillera, and David Cucurell for help with psychological assessment. The present project is supported by the Deutsche Forschungsgemeinschaft (SFB 779, TP A8, TP A5, TP B9), the "Pakt für Forschung und Innovation" of the Leibniz Society (WGL), and the State of Saxony-Anhalt.

genotype in the cycle of violence in maltreated children. Science 297, 851-854.

Dell'Acqua, M. L., Smith, K. E., Gorski, J. A., Horne, E. A., Gibson, E. S. and Gomez, L. L. (2006). Regulation of neuronal PKA signaling through AKAP targeting dynamics. Eur. J. Cell Biol. 85, 627-633.

Dolcos, F., and Mccarthy, G. (2006). Brain systems mediating cognitive interference by emotional distraction. J. Neurosci. 26, 2072-2079.

Efron, B., and Tibshirani, R. (1993). An Introduction to the Bootstrap. New York: Chapman \& Hall.

Egan, M. F., Goldberg, T. E., Kolachana, B. S., Callicott, J. H., Mazzanti, C. M., Straub, R. E., Goldman, D., and Weinberger, D. R. (2001). Effect of COMT Val108/158 Met genotype on frontal lobe function and risk for schizophrenia. Proc. Natl. Acad. Sci. U.S.A. 98, 6917-6922.

Egner, T., Etkin, A., Gale, S., and Hirsch, J. (2008). Dissociable neural systems resolve conflict from emotional versus nonemotional distracters. Cereb. Cortex 18, 1475-1484.

Eriksen, B., and Eriksen, C. (1974). Effects of noise letters upon the identification of a target letter in a nonsearch task. Percept. Psychophys. 16, 143-149.

Etkin, A., Egner, T., Peraza, D. M., Kandel, E. R., and Hirsch, J. (2006). Resolving emotional conflict: a role for the rostral anterior cingulate cortex in modulating activity in the amygdala. Neuron 51, 871-882.

Fava, M. (1997). Psychopharmacologic treatment of pathologic aggression. Psychiatr. Clin. North Am. 20, 427-451.

Fenske, M. J., and Eastwood, J. D. (2003). Modulation of focused attention by faces expressing emotion: evidence from flanker tasks. Emotion 3, 327-343.

Forbes, E. E., Brown, S. M., Kimak, M., Ferrell, R. E., Manuck, S. B., and Hariri, A. R. (2009). Genetic variation in components of dopamine neurotransmission impacts ventral 
striatal reactivity associated with impulsivity. Mol. Psychiatry 14, 60-70.

Fossati, A., Maffei, C., Acquarini, E., and Di Ceglie, A. (2003). Multigroup confirmatory component and factor analyses of the Italian version of the Aggression Questionnaire. Eur. J. Psychol. Assess. 19, 54-65.

Frank, B., Wiestler, M., Kropp, S., Hemminki, K., Spurdle, A. B., Sutter, C., Wappenschmidt, B., Chen, X., Beesley, J., Hopper, J. L., Australian Breast Cancer Family Study Investigators, Meindl, A., Kiechle, M., Slanger, T., Bugert, P., Schmutzler, R. K., Bartram, C. R., Flesch-Janys, D., Mutschelknauss, E., Ashton, K., Salazar, R., Webb, E., Hamann, U., Brauch, H., Justenhoven, C., Ko, Y. D., Brüning, T., Silva Idos, S., Johnson, N., Pharoah, P. P., Dunning, A. M., Pooley, K. A., ChangClaude, J., Easton, D. F., Peto, J., Houlston, R., Gene Environment Interaction and Breast Cancer in Germany Group, Kathleen Cuningham Foundation Consortium for Research into Familial Breast Cancer Investigators, Australian Ovarian Cancer Study Management Group, Chenevix-Trench, G., Fletcher, O., and Burwinkel, B. (2008). Association of a common AKAP9 variant with breast cancer risk: a collaborative analysis. J. Natl. Cancer Inst. 100, 437-4342.

Fraser, I. D., Cong, M., Kim, J., Rollins, E. N., Daaka, Y., Lefkowitz, R. J., and Scott, J. D. (2000). Assembly of an A kinase-anchoring proteinbeta(2)-adrenergic receptor complex facilitates receptor phosphorylation and signaling. Curr. Biol. 10, 409-412.

Friston, K. J., Fletcher, P., Josephs, O., Holmes, A., Rugg, M. D., and Turner, R. (1998). Event-related fMRI: Characterizing differential responses. Neuroimage 7, 30-40.

García-León, A., Reyes, G. A., Vila, J., Pérez, N., Robles, H., and Ramos, M. M. (2002). The Aggression Questionnaire: a validation study in student samples. Span. J. Psychol. 5, 45-53.

Gardner, L. A., Tavalin, S. J., Goehring, A. S., Scott, J. D., and Bahouth, S. W. (2006). AKAP79-mediated targeting of the cyclic AMP-dependent protein kinase to the betal-adrenergic receptor promotes recycling and functional resensitization of the receptor. J. Biol. Chem. 281, 33537-33553.

Gardner, L. A., Naren, A. P., and Bahouth, S. W. (2007). Assembly of an SAP97-AKAP79-cAMPdependent protein kinase scaffold at the type 1 PSD-95/DLG/ZO1 motif of the human beta(1)-adrenergic receptor generates a receptosome involved in receptor recycling and networking. J. Biol. Chem. 282, 5085-5099.

Haas, B. W., Omura, K., Constable, R. T., and Canli, T. (2006). Interference produced by emotional conflict associated with anterior cingulate activation. Cogn. Affect. Behav. Neurosci. 6, 152-156.

Hart, S. J., Green, S. R., Casp, M., and Belger, A. (2010). Emotional priming effects during Stroop task performance. Neuroimage 49, 2662-2670.

Heinz, A. J., Beck, A., MeyerLindenberg, A., Sterzer, P., and Heinz, A. (2011). Cognitive and neurobiological mechanisms of alcohol-related aggression. Nat. Rev. Neurosci. 12, 400-413.

Hess, C., Reif, A., Strobel, A., BoreattiHummer, A., Heine, M., Lesch, K. P., and Jacob, C. P. (2009). A functional dopamine-beta-hydroxylase gene promoter polymorphism is associated with impulsive personality styles, but not with affective disorders. J. Neural Transm. 116, 121-130.

Hinrichs, H., Scholz, M., Tempelmann, C., Woldorff, M. G., Dale, A. M., and Heinze, H. J. (2000). Deconvolution of event-related fMRI responses in fast-rate experimental designs: tracking amplitude variations. J. Cogn. Neurosci. 12(Suppl. 2), 76-89.

Horstmann, G., Borgstedt, K., and Heumann, M. (2006). Flanker effects with faces may depend on perceptual as well as emotional differences. Emotion 6, 28-39.

Kang, J. I., Namkoong, K., and Kim, S. J. (2008). Association of DRD4 and COMT polymorphisms with anger and forgiveness traits in healthy volunteers. Neurosci. Lett. 430, 252-257.

Kerns, J. G., Cohen, J. D., Macdonald, A. W. III., Cho, R. Y., Stenger, V. A., and Carter, C. S. (2004). Anterior cingulate conflict monitoring and adjustments in control. Science 303, 1023-1026.

Krämer, U. M., Cunillera, T., Camara, E., Marco-Pallares, J., Cucurell, D., Nager, W., Bauer, P., Schule, R., Schols, L., Rodriguez-Fornells, A., and Munte, T. F. (2007). The impact of catechol-O-methyltransferase and dopamine D4 receptor genotypes on neurophysiological markers of performance monitoring. J. Neurosci. 27, 14190-14198.

Kulikova, M. A., Maluchenko, N. V., Timofeeva, M. A., Shlepzova, V. A., Schegolkova, J. V., Sysoeva, O. V., Ivanitsky, A. M., and Tonevitsky, A. G. (2008). Effect of functional
catechol-O-methyltransferase Val158Met polymorphism physical aggression. Bull. Exp. Biol. Med. 145, 62-64.

Larson, M. J., Perlstein, W. M., Stigge-Kaufman, D., Kelly, K. G., and Dotson, V. M. (2006). Affective context-induced modulation of the error-related negativity. Neuroreport 17, 329-333.

Lundqvist, D., Flykt, A., and Öhman, A. (1998). The Karolinska Directed Emotional Faces - KDEF, CD ROM from Psychology Section, Department of Clinical Neuroscience, Karolinska Institutet, Stockholm.

Mansouri, F. A., Tanaka, K., and Buckley, M. J. (2009). Conflict-induced behavioural adjustment: a clue to the executive functions of the prefrontal cortex. Nat. Rev. Neurosci. 10, 141-152.

Meesters, C., Muris, P., Bosma, H. Schouten, E., and Beuving, S. (1996). Psychometric evaluation of the Dutch version of the Aggression Questionnaire. Behav. Res. Ther. 34, 839-843.

Meyer-Lindenberg, A., Buckholtz, J. W. Kolachana, B., Hariri, R. A., Pezawas, L., Blasi, G., Wabnitz, A., Honea, R. Verchinski, B., Callicott, J. H., Egan, M., Mattay, V., and Weinberger, D. R. (2006). Neural mechanisms of genetic risk for impulsivity and violence in humans. Proc. Natl. Acad. Sci. U.S.A. 103, 6269-6274.

Meyer-Lindenberg, A., and Weinberger, D. R. (2006). Intermediate phenotypes and genetic mechanisms of psychiatric disorders. Nat. Rev. Neurosci. 7, 818-827.

Mier, D., Kirsch, P., and MeyerLindenberg, A. (2010). Neural substrates of pleiotropic action of genetic variation in COMT: a meta-analysis. Mol. Psychiatry 15, 918-927.

Moita, M. A., Lamprecht, R., Nader, K. and Ledoux, J. E. (2002). A-kinase anchoring proteins in amygdala are involved in auditory fear memory. Nat. Neurosci. 5, 837-838.

Nakano, K. (2001). Psychometric evaluation on the Japanese adaptation of the Aggression Questionnaire. Behav. Res. Ther. 39, 853-858.

Niedenthal, P. M. (2007). Embodying emotion. Science 316, 1002-1005.

Nijholt, I. M., Ostroveanu, A., Scheper, W. A., Penke, B., Luiten, P. G., Van Der Zee, E. A., and Eisel, U. L. (2008). Inhibition of PKA anchoring to Akinase anchoring proteins impairs consolidation and facilitates extinction of contextual fear memories. Neurobiol. Learn. Mem. 90, 223-229. Ostroveanu, A., Van Der Zee, E. A., Dolga, A. M., Luiten, P. G., Eisel,
U. L., and Nijholt, I. M. (2007). Akinase anchoring protein 150 in the mouse brain is concentrated in areas involved in learning and memory. Brain Res. 1145, 97-107.

Panksepp, J. (2006). Emotional endophenotypes in evolutionary psychiatry. Prog. Neuropsychopharmacol. Biol. Psychiatry 30, 774-784.

Perlis, R. H., Purcell, S., Fagerness, J., Cusin, C., Yamaki, L., Fava, M., and Smoller, J. W. (2007). Clinical and genetic dissection of anger expression and CREB1 polymorphisms in major depressive disorder. Biol. Psychiatry 62, 536-540.

Pessoa, L. (2008). On the relationship between emotion and cognition. Nat. Rev. Neurosci. 9, 148-158.

Popova, N. K. (2008). From gene to aggressive behavior: the role of brain serotonin. Neurosci. Behav. Physiol. 38, 471-475.

Ramírez, J. M., Fujihara, T., and van Goozen, S. (2001). Cultural and gender differences in anger and aggression: a comparison between Japanese, Dutch, and Spanish students. J. Soc. Psychol. 141, 119-121.

Ridderinkhof, K. R., Ullsperger, M. Crone, E. A., and Nieuwenhuis, S. (2004). The role of the medial frontal cortex in cognitive control. Science 306, 443-447.

Sandoz, G., Thummler, S., Duprat, F., Feliciangeli, S., Vinh, J., Escoubas, P., Guy, N., Lazdunski, M., and Lesage, F. (2006). AKAP150, a switch to convert mechano-, $\mathrm{pH}$ - and arachidonic acid-sensitive TREK $\mathrm{K}(+)$ channels into open leak channels. EMBO J. 25, 5864-5872.

Schott, B. H., Seidenbecher, C. I., Fenker, D. B., Lauer, C. J., Bunzeck, N., Bernstein, H. G., Tischmeyer, W., Gundelfinger, E. D., Heinze, H. J., and Düzel, E. (2006). The dopaminergic midbrain participates in human episodic memory formation: evidence from genetic imaging. J. Neurosci. 26, 1407-1417.

Schwenkmezger, P., and Hodapp, V. (1991). A questionnaire for assessing anger and the expression of anger. $Z$. Klin. Psychol. Psychiatr. Psychother. 39, 63-68.

Seidenbecher, C. I., Smalla, K.-H., Fischer, N., Gundelfinger, E. D., and Kreutz, M. R. (2002). Brevican isoforms associate with neural membranes. J. Neurochem. 83, 738-746.

Sik, A., Gulacsi, A., Lai, Y., Doyle, W. K., Pacia, S., Mody, I., and Freund, T. F. (2000). Localization of the A kinase anchoring protein AKAP79 in the human hippocampus. Eur. J. Neurosci. 12, 1155-1164. 
Spielberger, C. D. (1991). StateTrait Anger Expression Inventory: STAXI Professional Manual. Psychological Assessment Resources, Florida.

Sutrala, S. R., Goossens, D., Williams, N. M., Heyrman, L., Adolfsson, R., Norton, N., Buckland, P. R., and DelFavero, J. (2007). Gene copy number variation in schizophrenia. Schizophr. Res. 96, 93-99.

Swayze, R. D., Lise, M. F., Levinson, J. N., Phillips, A., and ElHusseini, A. (2004). Modulation of dopamine mediated phosphorylation of AMPA receptors by PSD-95 and AKAP79/150. Neuropharmacology 47, 764-778.

Tabachnick, B. G., and Fidell, L. S. (1983). Using Multivariate Statistics, Chapter 9. New York: Harper \& Row.

Tunquist, B. J., Hoshi, N., Guire, E. S., Zhang, F., Mullendorff, K., Langeberg, L. K., Raber, J., and Scott, J. D. (2008). Loss of AKAP150 perturbs distinct neuronal processes in mice. Proc. Natl. Acad. Sci. U.S.A. 105, 12557-12562.

Ulfig, N., Neudorfer, F., and Bohl, J. (2001). Development-related expression of AKAP79 in the striatal compartments of the human brain. Cells Tissues Organs 168, 319-329.

Ulfig, N., Setzer, M., and Bohl, J. (2003). Ontogeny of the human amygdala. Ann. N. Y. Acad. Sci. 985, 22-33.

Van Honk, J., Tuiten, A., De Haan, E., Ven Den Hout, M., and Stam, H. (2001). Attentional biases for angry faces: relationships to trait anger and anxiety. Cogn. Emot. 15, 279-297.

Von Collani, G., and Werner, R. (2005). Self-related and motivational constructs as determinants of aggression. An analysis and validation of a German version of the Buss-Perry Aggression Questionnaire. Pers. Individ. Dif. 38, 1631-1643.

Wilson, G. M., Flibotte, S., Chopra, V., Melnyk, B. L., Honer, W. G., and Holt, R. A. (2006). DNA copy-number analysis in bipolar disorder and schizophrenia reveals aberrations in genes involved in glutamate signaling. Hum. Mol. Genet. 15, 743-749.

Wimber, M., Schott, B. H., Wendler, F., Seidenbecher, C. I., Behnisch, G., Macharadze, T., Bäuml, K. H., and Richardson-Klavehn, A. (2011). Prefrontal dopamine and the dynamic control of human longterm memory. Transl. Psychiatr. $1, \mathrm{e} 15$.

Wiswede, D., Münte, T. F., Goschke, T., and Rüsseler, J. (2009). Modulation of the error-related negativity by induction of short-term negative affect. Neuropsychologia 47 , 83-90.

Conflict of Interest Statement: The authors declare that the research was conducted in the absence of any commercial or financial relationships that could be construed as a potential conflict of interest.
Received: 01 July 2011; accepted: 13 December 2011; published online: 29 December 2011.

Citation: Richter S, Gorny X, MarcoPallares J, Krämer UM, Machts J, Barman A, Bernstein H-G, Schüle R, Schöls L, Rodriguez-Fornells A, Reissner C, Wüstenberg T, Heinze H-J, Gundelfinger ED, Düzel E, Münte TF, Seidenbecher CI and Schott BH (2011) A potential role for a genetic variation of AKAP5 in human aggression and anger control. Front. Hum. Neurosci. 5:175. doi: 10.3389/fnhum.2011.00175

Copyright (c) 2011 Richter, Gorny, Marco-Pallares, Krämer, Machts, Barman, Bernstein, Schüle, Schöls, Rodriguez-Fornells, Reissner, Wüstenberg, Heinze, Gundelfinger, Düzel, Münte, Seidenbecher and Schott. This is an open-access article distributed under the terms of the Creative Commons Attribution Non Commercial License, which permits non-commercial use, distribution, and reproduction in other forums, provided the original authors and source are credited. 